\title{
Measurements of low energy observables, elastic $p p$ interac- tions and exclusive production in proton-proton collisions with the ATLAS Detector
}

\author{
P. J. Bussey ${ }^{1, a}$ \\ On behalf of the ATLAS Collaboration \\ ${ }^{1}$ SUPA, School of Physics and Astronomy \\ University of Glasgow \\ Glasgow G12 8QQ, U.K.
}

\begin{abstract}
A summary is given of recent ATLAS results at the LHC, covering a number of areas that reflect the Collaboration's work in low energy physical observables in multiparticle events, elastic photon-photon scattering, proton-proton scattering and the tagging of diffractive events.
\end{abstract}

\section{Introduction}

A variety of processes can be studied at the LHC that involve relatively soft final states. This talk presents results from a selection of different measurements of this kind, with the aim of illustrating the wide range of physics that can be performed using the ATLAS detector [1].

\section{Single-particle distributions at low transverse momentum}

ATLAS have repeated earlier studies of single-particle distributions by measurements at the LHC's highest energy of $\sqrt{s}=13 \mathrm{GeV}$ [2]. The results can be used to compare different tunes of Monte Carlo (MC) generators, in particular PутніA, and a selection of the distributions is shown in Fig. 1 The number of charged particles with $p_{T}>100 \mathrm{MeV}$ per event is well described at the 10-20\% level by several tunes and models, but above $120 \mathrm{GeV}$ the predictions diverge, and only Epos LHC is satisfactory of those considered here. Averaged over all transverse momentum $\left(p_{T}\right)$ values, the mean number of charged particles per unit of pseudorapidity is described worst by PYтнIA 8 A2, but the agreements are still all good to $7 \%$. None of the models succeeds very well at the lowest $p_{T}$ values, although Epos LHC is best. When the mean $p_{T}$ of the particles is plotted as a function of the number of charged particles in the event, it is apparent that QGSJET 11-04 does not model this variable properly. Of the other models, only Epos LHC is satisfactory.

Similar conclusions can be drawn when the present data are compared with previous measurements, as illustrated in Fig. 2. The general shape of the charge multiplicity distribution in pseudorapidity is well described overall as a function of LHC beam energy for several different plotted conditions. 

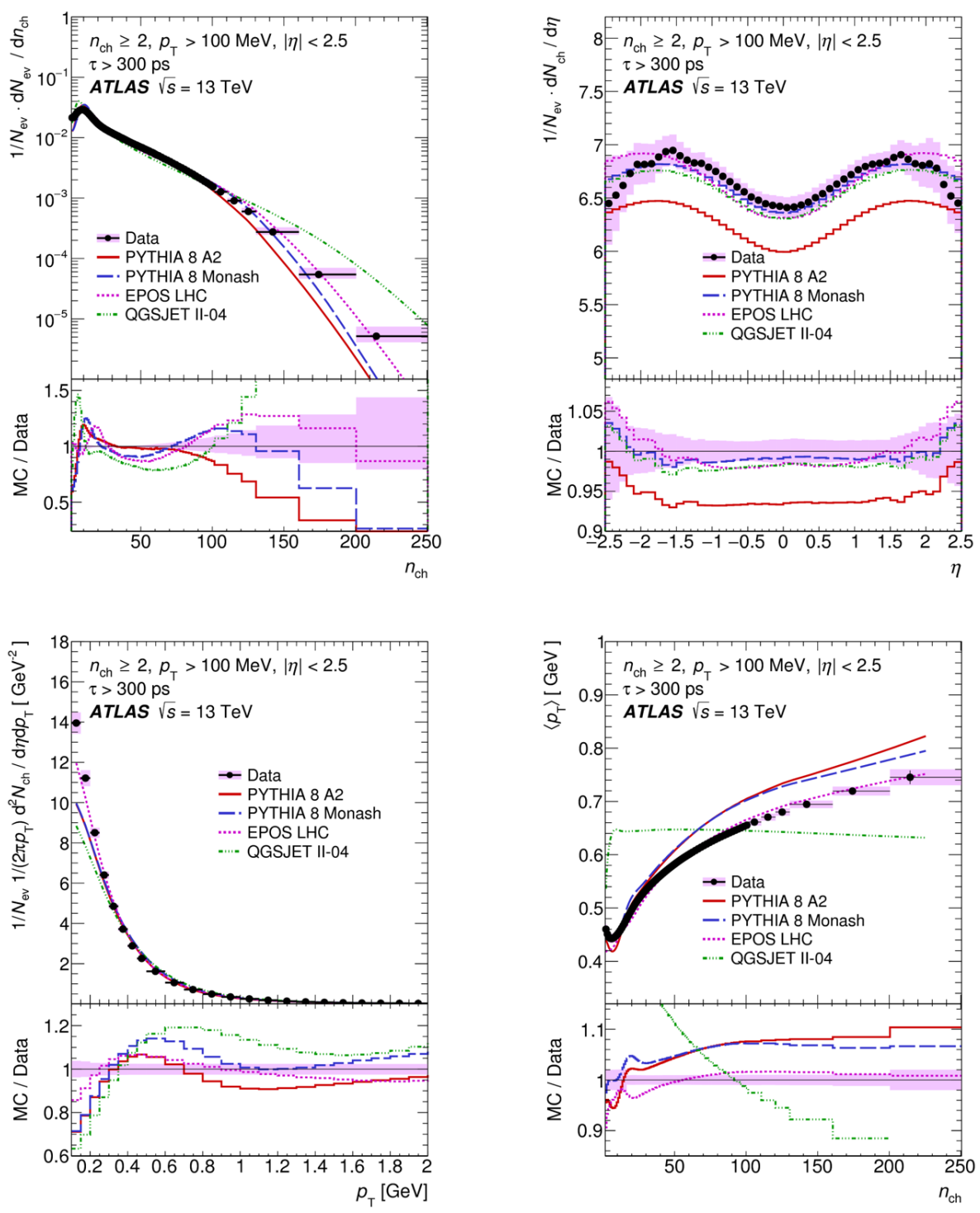

Figure 1. Distributions of centrally produced charged particles in ATLAS with transverse momentum $p_{T}$ greater than $100 \mathrm{MeV} / c$ [2]. Normalised cross sections are shown as a function of the number of tracks, the pseudorapidity, and the $p_{T}$ of the tracks, the last of these scaled by $1 / p_{T}$. In the final plot, the mean $p_{T}$ is shown as $\mathrm{s}$ function of the charged particle multiplicity.

\footnotetext{
ae-mail: peter.bussey@glasgow.ac.uk
} 


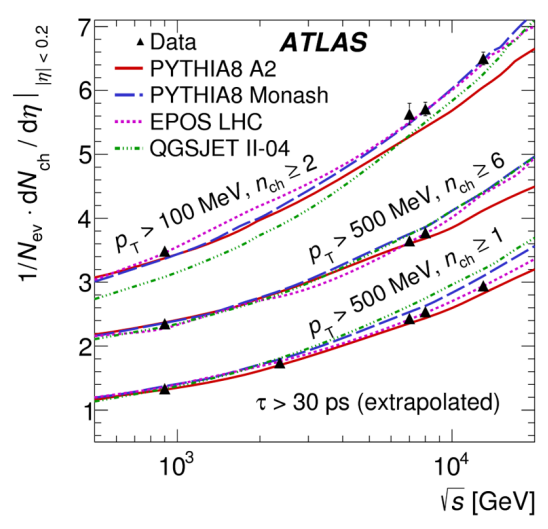

Figure 2. Mean density in pseudorapidity of centrally produced tracks in ATLAS, as a function of $\sqrt{s}$, for tracks with a given minimum $p_{T}$ value, compared to several MC models and tunes [2].

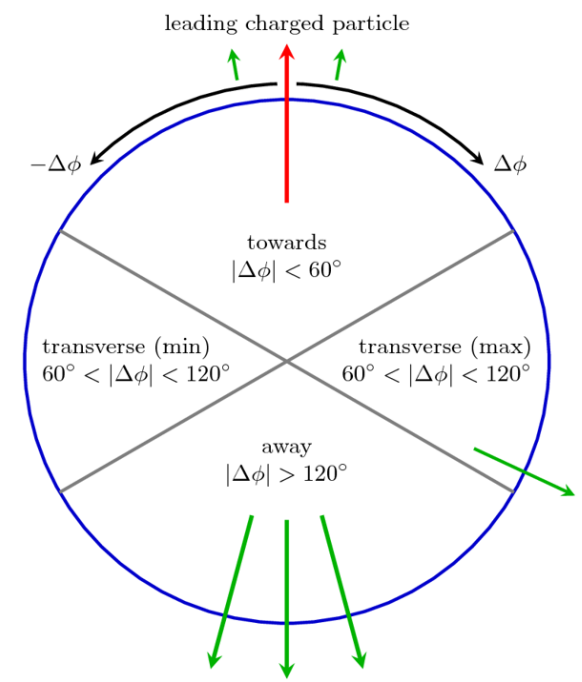

Figure 3. Schematic diagram of the azimuthal structure of QCD scattering events at the LHC [3].

\section{Measurement of distributions sensitive to the underlying event in hard scattering processes}

In hard parton scattering processes, the underlying event (UE) is understood as whatever is not associated with the hard scatter. In a recent study [3] ATLAS have measured properties of the UE. The structure of the events in the azimuthal plane is shown schematically in Fig. 3. No jet analysis is performed, but the leading scattered charged particle is identified, and regions of the event are defined 
relative to this. The "towards" and "away" regions are defined as those that cover azimuths within $60^{\circ}$ of the leading particle and above $120^{\circ}$, respectively. The transverse regions, which are those where the UE features, have azimuths between the towards and away regions. In the two transverse regions, the summed $p_{T}$ of the scattered particles is calculated and the trans-max and trans-min regions are those with the greater and lesser value of this quantity.

Figure 4 shows that the $p_{T}$ distribution of the leading charged particle is well described by several MC tunes and models over values between 1 and 30 GeV. The Pythia 8 A14 and Herwig 7 models are less good than the others illustrated. The description of the UE can be affected by this quantity, and a poor description may be indicative of problems with the model.

Figure 5 shows a comparison between the present measurements at $\sqrt{s}=13 \mathrm{TeV}$ and those taken at lower LHC energies. The total summed $p_{T}$ in the transverse region and the number of charged tracks in this region are plotted as functions of the $p_{T}$ of the leading charged particle, and it is seen that at all LHC energies, the plotted quantities form a plateau-like shoulder once the leading $p_{T}$ reaches a value of approximately $5 \mathrm{GeV}$, which does not vary much with $\sqrt{s}$, showing that the UE has some fairly well-defined properties. However the value of the plotted quantity varies strongly with $\sqrt{s}$, showing that the available energy is shared with both the hard scatter and the UE.

The mean charged particle $p_{T}$ per unit of pseudorapdity and azimuth at $\sqrt{s}=13 \mathrm{TeV}$ is also plotted in Fig. 6 for the trans-max region. Fig. 6 also shows the charged multiplicty, in both cases compared to several MC tunes and models as before. As a function of the $p_{T}$ of the leading particle, all the models perform well, but HerwiG is the most successful. All the models give a good description of the mean $p_{T}$ as a function of transverse charged particle multiplicity. The corresponding plots and comparisons for the trans-min region are similar.

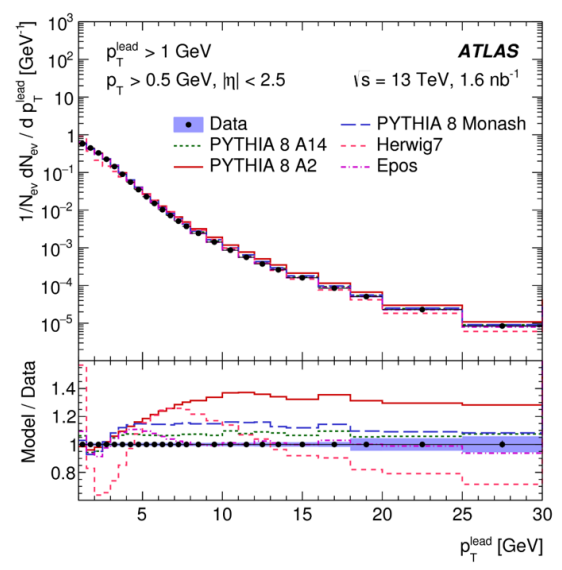

Figure 4. Normalised differential cross section of the leading charged particle in hadronic scattering events in ATLAS, compared to a selection of MC models and tunes [3]. 

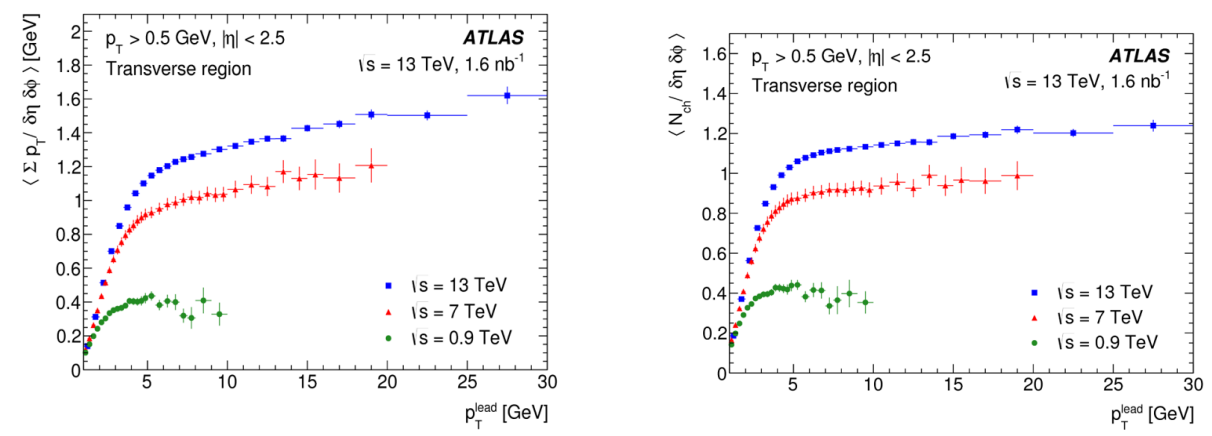

Figure 5. Distributions in the summed $p_{T}$ and the number of charged tracks in the transverse azimuthal region, per unit pseudorapdity and azimuth, as functions of the $p_{T}$ of the leading charged track in the event, for different values of LHC collision energy [3].
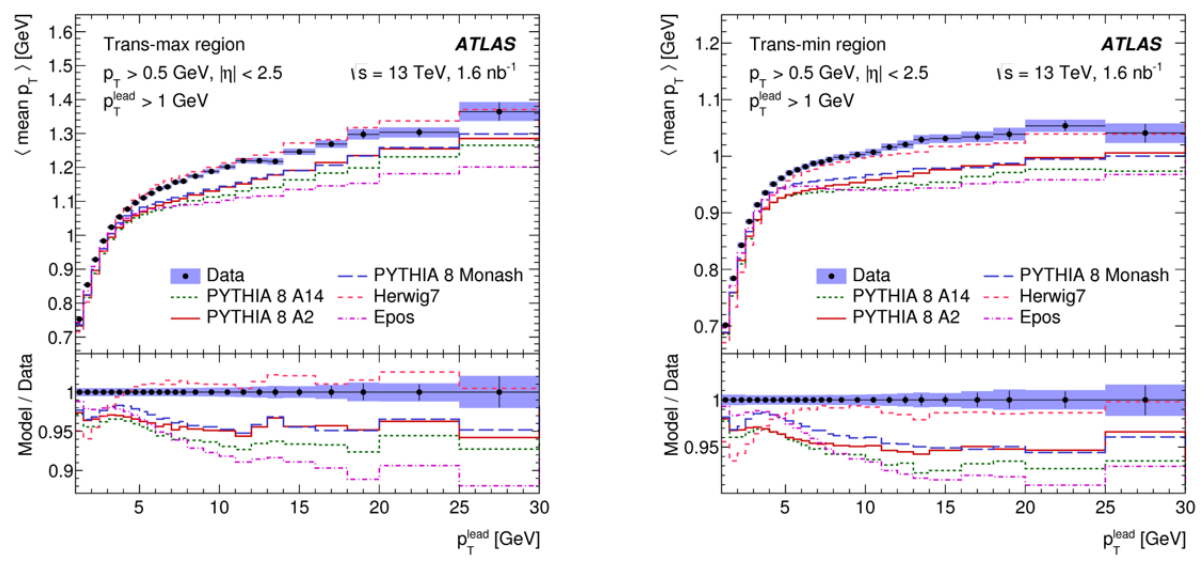

Figure 6. Distributions of the mean $p_{T}$ of centrally produced charged particles in the trans-max region, as functions of the leading particle $p_{T}$ and the charged multiplicity in the transverse region [3].

\section{Study of ordered hadron chains with the ATLAS detector}

In MC simulations based on the Lund string model, such as PyтніA, the colour string is postulated as a linear object that splits along its length. More complex types of colour connection are in principle possible, and ATLAS have studied a proposal based on a helical string structure. In this proposal [4], new features may in principle be observed, and a new test has been performed by ATLAS using $7 \mathrm{TeV}$ data. Minimum-bias events are selected and triplets of charged tracks are studied by finding the likesign track pair with the smallest value of $Q^{2}=-\left(p_{i}-p_{j}\right)^{2}$ and then identifying the third opposite-sign track which minimises the overall triplet mass. A Dalitz plot is then constructed in terms of the parameters $X=\sqrt{3}\left(T_{0}-T_{2}\right) / \sum T$ and $Y=3 T_{1} / \sum T-1$ where $T$ is kinetic energy and the like-sign particles are labelled 0,2 . 
Figure 7 shows on the left the Dalitz plot that is obtained using events generated by PүтнIA; a broad, featureless plateau is seen. HerwIG gives rise to a structure that is featureless in the $X$ direction but shows a gradual, smooth enhancement in the $Y$ direction. On the right, while Epos shows a broad central enhancement in $Y$. The Dalitz plot in $X, Y$ obtained from the data [5], by contrast, exhibits indications of narrow structures at low $X$ and high $Y$, incompatible with the MC models that have been studied. Also shown in the Figure are some indications of the type of structure that the helical string model predicts. These are in qualitative agreement with the data and give support for further investigation of this type of model.
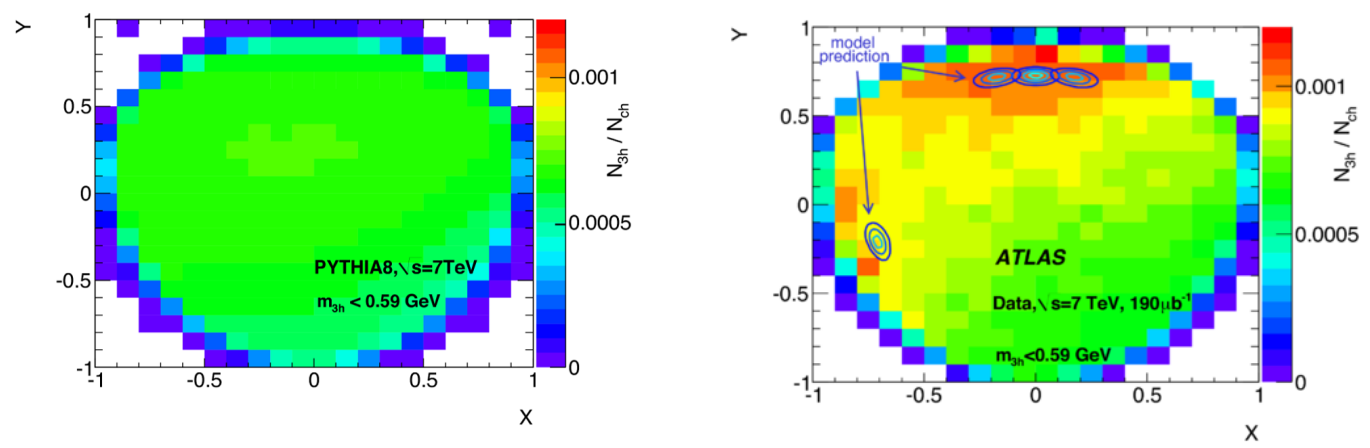

Figure 7. (Left) Dalitz plot of variables $X$ and $Y$, as defined in the text, obtained using PYтніA 84 C. (Right) Dalitz plot from data, on which are superposed model predictions involving $\pi^{+} \pi^{-} \pi^{0} \pi^{+}$combinations with unobserved $\pi^{0}$, at $X \approx-0.7$, and triplets of charged pions with and without a further unobserved particle, at $Y \approx 0.7$ [5].

\section{Measurement of exclusive two-photon production of muon pairs}

At LHC energies, high-energy photons are radiated sufficiently strongly from the incoming protons for photon-photon processes to be measurable. The simplest of the processes to measure is the exclusive production of muon pairs, since these are not produced by hadronic mechanisms. ATLAS have performed a measurement of the process $\gamma \gamma \rightarrow \mu^{+} \mu^{-}[6]$ in which the two exchanged photons are radiated from the incoming protons, which emerge scattered through a small angle and are not observed. Accompanying the exclusive mechanism are processes in which one or both of the protons emerge in a dissociated state (single or double dissociative processes); the products of the proton dissociation are usually unobserved, but the dynamics of the process may be affected.

To restrict the measurement to the electromagnetic production of muon pairs, the inclusion of $Z \rightarrow \mu^{+} \mu^{-}$events is avoided by selecting events that contain muon pairs with mass in the range $12-70 \mathrm{GeV}$. The primary vertex must be isolated, with a requirement that the total $p_{T}$ of the muon pairs must be less than $1.5 \mathrm{GeV}$ in order to suppress single-dissociative events. The acoplanarity of the muon pairs after these selections is shown in Fig. 8 and shows that the observed process can be well modelled in terms of exclusive and single dissociative events, with a very small admixture of double dissociative events. Other backgrounds are negligible. The figure also shows the measured differential cross section in the dimuon mass. An equivalent-photon-approximation model (EPA) describes the data well, and better than the more elaborate SuperChic2 model. 

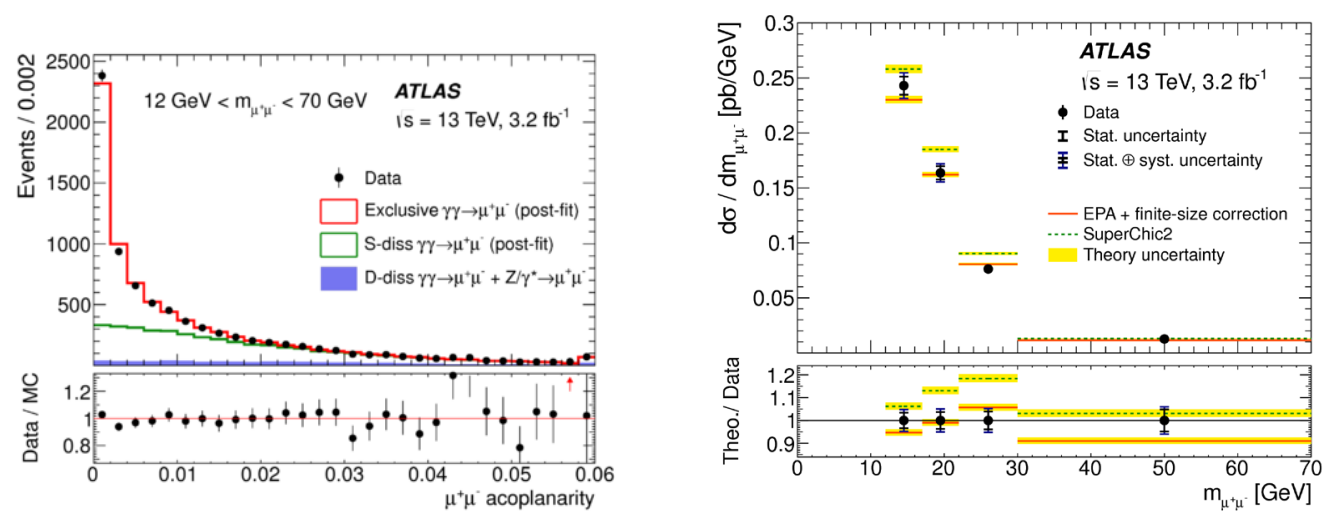

Figure 8. (Left) Muon pair acoplanarity in events with just an observed muon pair, modelled as the sum of the exclusive process and the single and double proton dissociative processes. (Right) Differential cross section in the muon pair mass for exclusive events, compared to the EPA and SuperChic models [6].

\section{Total $p p$ scattering cross section}

The most basic measurement to be made in particle-particle scattering is the total scattering cross section. This has been measured by ATLAS at $\sqrt{s}=8 \mathrm{TeV}$ [8] by means of the ALFA sub-detector [7], which consists of arrays of scintillating fibre detectors mounted in Roman Pots either side of the two emerging proton beams at distances of 237 and $241 \mathrm{~m}$ downstream of the main detector (Fig. 9). Coincidences between counts in these systems measure elastic proton-proton scattering events. Special beam conditions are required to enable the elastic scattered protons to reach the detectors down to scattering angles that are as possible with negligible pile-up.

Figure 10 shows the measured differential cross sections as a function of $-t$. A fit to the data at low $t$ enables the total cross section to be evaluated though the Optical Theorem, by means of

$$
\sigma_{\mathrm{tot}}^{2}=\frac{16 \pi(\hbar c)^{2}}{1+\rho^{2}} \frac{\mathrm{d} \sigma_{\mathrm{el}}}{\mathrm{d} t}
$$

where the measured cross section is extrapolated to $t=0$. It is also possible to extract from the measurements the total elastic cross section and hence the total inelastic cross section, whose measurement by this technique has advantages over its measurement by triggering on minimum-bias events in the central detector. These total cross sections are also shown in the figure in comparison with measurements over a wide range of proton-proton energies, including earlier results from ATLAS at $\sqrt{s}=7 \mathrm{TeV}$ [9]. The picture is consistent and there is satisfactory agreement with the results from TOTEM at the LHC. 


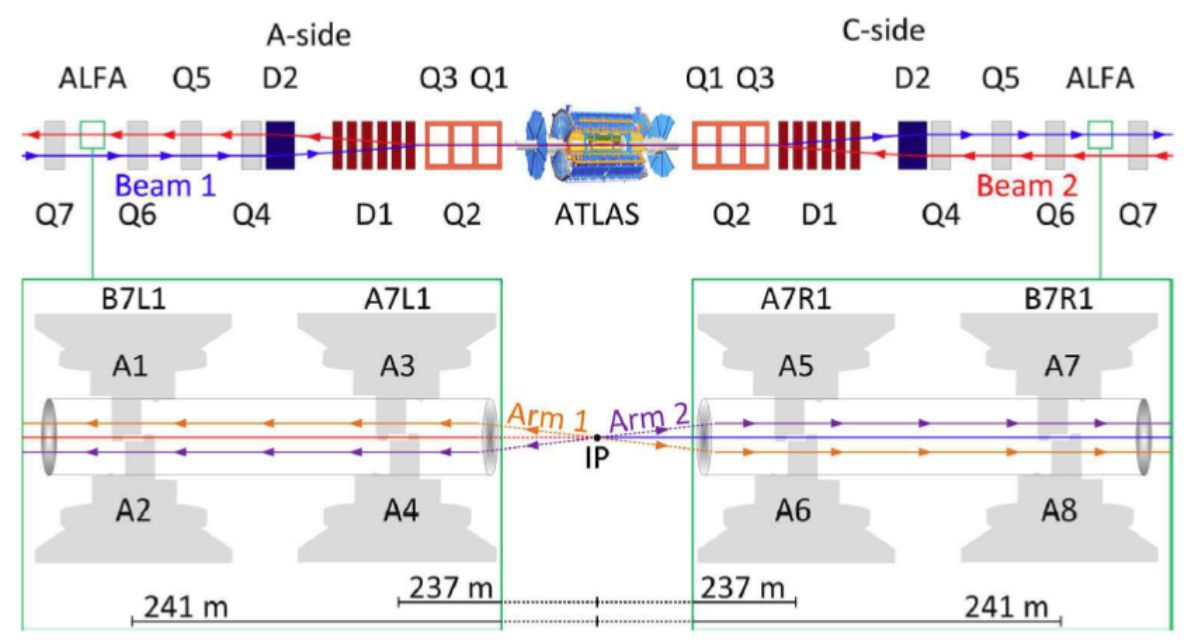

Figure 9. Beam configuration in the region of the ATLAS detector showing the location of the ALFA subdetectors [7].

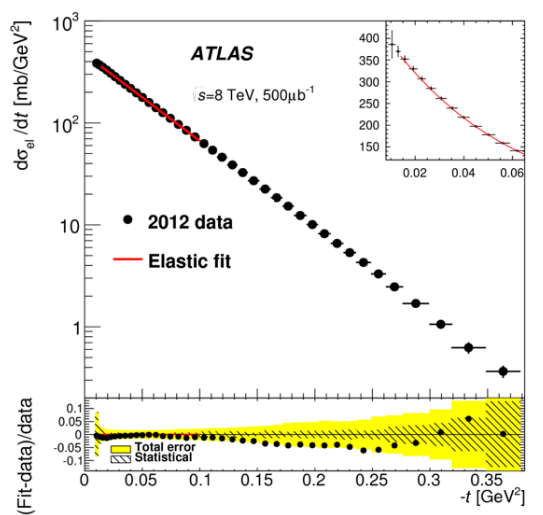

(b)

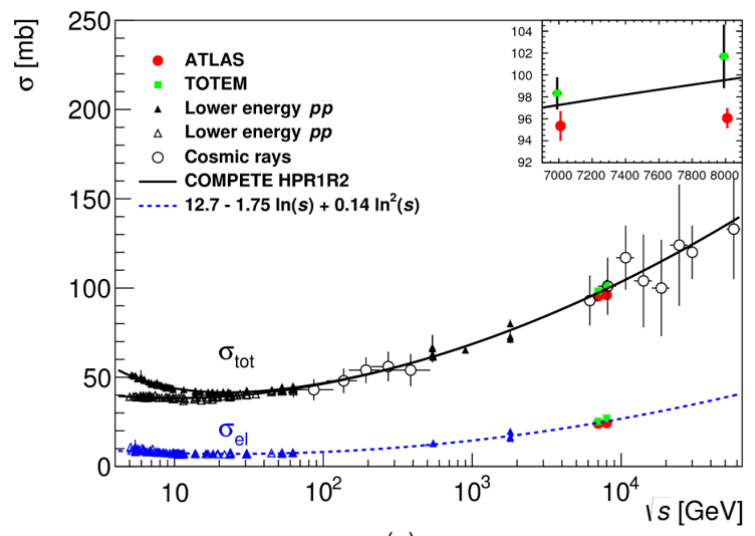

(a)

Figure 10. (Left) Differential cross section in $t$ of the elastic scattering of two protons in ATLAS at $\sqrt{s}=$ $8 \mathrm{TeV}$ [8]. (Right) Measured total and elastic cross sections in comparison with results from other measurements. [8,9]

\section{The ATLAS Forward Proton system}

The ATLAS Forward Proton sub-detectors (AFP) [10] have now been installed and resemble the ALFA system in that they detect forward scattered protons. They are located 205-217 $\mathrm{m}$ downstream of ATLAS in both emerging beamlines, as seen in Fig. 11. The protons measured in AFP have lost energy in a diffractive scatter in the main detector, and the bending magnets in the beamline act as a spectrometer to measure the energy of the forward diffractive proton, so as to be able to 


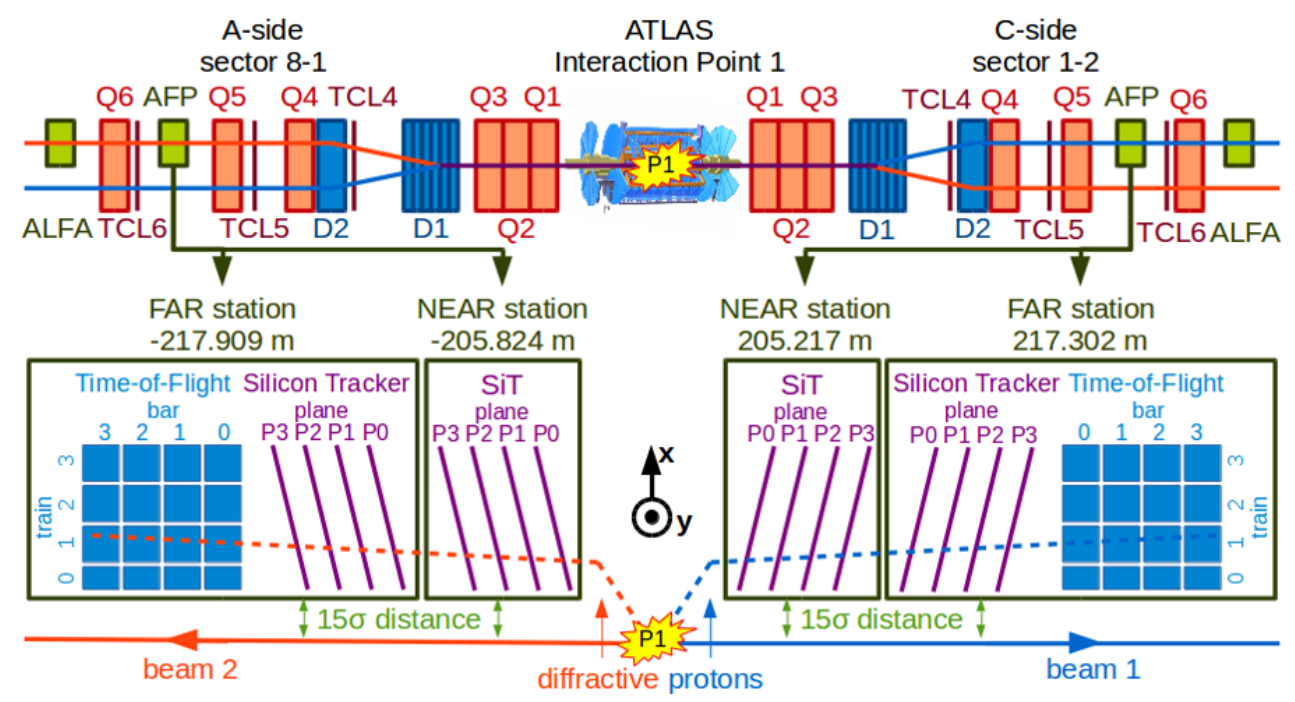

Figure 11. Beam configuration in the region of the ATLAS detector showing the location of the AFP subdetectors and a schematic representation of their layout [10].

correlate this with the energy measured in the central detector. The AFP system consists of silicon tracking detectors, backed by a time-of-flight system that is intended to help identify the position of the scattering event relative to the nominal interaction point and enable pile-up events to be correctly distinguished.

First results have now been obtained. Figure 12 shows the distribution (red) of the fractional proton energy loss calculated from events recorded by the ATLAS minimum-bias trigger [11]. This is compared (suitably normalised) with a similar distribution (black) obtained with events using the AFP trigger. An upper peak resembles the minimum-bias peak and indicates random coincidences between the forward proton and the centrally detected event. A lower peak is distinct from this, and indicates that a coincidence is indeed measured between the AFP-detected proton and an event in the central detector.

\section{Conclusions}

We have seen here a small but broad selection of lower-energy physics results, both final and under development, that have been obtained using the ATLAS detector. They indicate once again the exceptionally wide range of physics that can be studied at the LHC. 


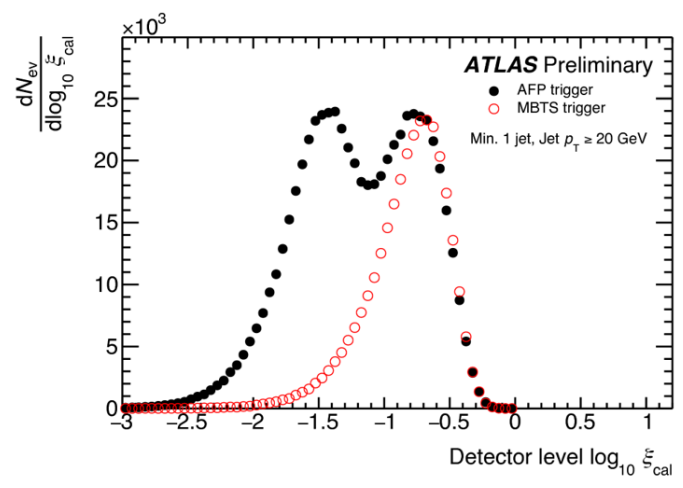

Figure 12. Calculated fractional loss of energy of protons that undergo minimum-bias triggered events (red) and AFP-triggered events (black) [11].

\section{References}

[1] ATLAS Collaboration, J. Inst. 3 S08003 (2008)

[2] ATLAS Collaboration, Eur. Phys. J. C 502 (2016)

[3] ATLAS Collaboration, JHEP 03157 (2017)

[4] S. Todorova, Phys. Rev. D89 015002 (2014)

[5] ATLAS Collaboration, arXiv: 1709.07384

[6] ATLAS Collaboration, arXiv: 1708.04053

[7] ATLAS Collaboration, JINST 11 P11013 (2016)

[8] ATLAS Collaboration, Phys. Lett. B 761158 (2016)

[9] ATLAS Collaboration, Nucl. Phys. B 889486 (2014)

[10] ATLAS Collaboration, ATL-TDR-024

[11] ATLAS Collaboration, ATL-PHYS-PUB-2017-012 